\title{
Critical Temperature of Weakly Interacting Dipolar Condensates
}

\author{
Konstantin Glaum ${ }^{1}$, Axel Pelster ${ }^{2}$, Hagen Kleinert ${ }^{1}$, and Tilman Pfau $^{3}$ \\ ${ }^{1}$ Fachbereich Physik, Freie Universität Berlin, Arnimallee 14, 14195 Berlin, Germany \\ ${ }^{2}$ Fachbereich Physik, Universität Duisburg-Essen, Lotharstraße 1, 47048 Duisburg, Germany \\ ${ }^{3}$ 5. Physikalisches Institut, Universität Stuttgart, Pfaffenwaldring 57, 70569 Stuttgart, Germany
}

(Dated: June 27, 2018)

\begin{abstract}
We calculate perturbatively the effect of a dipolar interaction upon the Bose-Einstein condensation temperature. This dipolar shift depends on the angle between the symmetry axes of the trap and the aligned atomic dipole moments, and is extremal for parallel or orthogonal orientations, respectively. The difference of both critical temperatures exhibits most clearly the dipole-dipole interaction and can be enhanced by increasing both the number of atoms and the anisotropy of the trap. Applying our results to chromium atoms, which have a large magnetic dipole moment, shows that this dipolar shift of the critical temperature could be measured in the ongoing Stuttgart experiment.
\end{abstract}

PACS numbers: 03.75.Hh, 31.15.Gy, 51.30.+i

Ultracold atomic quantum gases are many-body systems in which macroscopic quantum phenomena can be studied experimentally over a wide range of controllable interactions [1-3]. For the original alkali atomic BoseEinstein condensates (BECs) it has been sufficient to describe the dominant two-particle interaction by a local isotropic contact potential. Recently, a new type of nonlocal anisotropic interaction has been made accessible to detailed study by the formation of a BEC in a dipolar quantum gas of ${ }^{52} \mathrm{Cr}$ [4]. Chromium atoms possess a magnetic dipole moment of $6 m_{B}$, where $m_{B}$ is the Bohr magneton, i.e. it is around six times larger than those of alkali atoms. It stands for a whole class of high spin atoms like rare earth atoms with large magnetic dipole moments (Dy $\left[10 m_{B}\right]$, Ho $\left[9 m_{B}\right]$, Eu $\left[7 m_{B}\right]$, Tb [10 $\left.m_{B}\right], \operatorname{Er}\left[7 m_{B}\right]$, Mo $\left[6 m_{B}\right], \operatorname{Mn}\left[5 m_{B}\right], \operatorname{Tm}\left[4 m_{B}\right]$, $\left.\operatorname{Pr}\left[3.3 m_{B}\right]\right)$. Note that these elements have been already cooled by buffer gas and evaporative cooling techniques down to $\mathrm{mK}$ temperatures [5]. Besides that $\operatorname{Er}\left[7 m_{B}\right]$ has recently been laser cooled [6]. Other many-body systems with dipolar interactions are, for instance, Rydberg atoms $[7,8]$ or atomic condensates where a strong electric field induces electric dipole moments of the order of $10^{-2}$ Debye [9]. Body-centered dipole moments in heteronuclear molecules are much larger with typical values of 1 Debye, so their dipolar effects could be a few hundred times stronger than those of chromium atoms [10, 11]. Such a gas of ultracold heteronuclear molecules is produced either by sophisticated cooling and trapping techniques $[12,13]$ or by photoassociation [14-16]. For all those dipolar systems the total two-particle interaction potential is modelled by

$$
\begin{aligned}
V^{(\mathrm{int})}\left(\mathbf{x}-\mathbf{x}^{\prime}\right) & =\frac{4 \pi \hbar^{2} a}{M} \delta\left(\mathbf{x}-\mathbf{x}^{\prime}\right) \\
- & \frac{\mu_{0}}{4 \pi}\left\{\frac{3\left[\mathbf{m}\left(\mathbf{x}-\mathbf{x}^{\prime}\right)\right]^{2}}{\left|\mathbf{x}-\mathbf{x}^{\prime}\right|^{5}}-\frac{\mathbf{m}^{2}}{\left|\mathbf{x}-\mathbf{x}^{\prime}\right|^{3}}\right\} .
\end{aligned}
$$

Here $\mathbf{m}$ denotes the magnetic (electric) dipole moment of the atoms, $\mu_{0}$ stands for the magnetic field constant (the reciprocal electric field constant), and $M$ is the atomic mass. A more general pseudopotential for anisotropic interactions has recently been introduced in Refs. [17, 18] which is nonlocal in momentum space. Since there exists up to now no experimental evidence for any dipolar shape resonance, where such a more general pseudopotential could be relevant, our model (1) is valid for all current and many future experimental situations.

The dimensionless measure of the strength of the dipoledipole interaction with respect to the s-wave scattering is $\epsilon_{D D}=\mu_{0} m^{2} M /\left(12 \pi \hbar^{2} a\right)$ [19]. For the ${ }^{52} \mathrm{Cr}$ condensate it has the value $\epsilon_{D D}=0.144$ [20], so the magnetic dipolar interaction represents only a small correction to the contact interaction. Nevertheless, it has interesting consequences due to its anisotropy, as has been observed in a recent expansion experiment [21]. Furthermore, the magnetic dipole-dipole interaction can be varied within a limited range with the help of rotating magnetic fields as proposed by Ref. [22]. Combining this technique with the now known 14 Feshbach resonances of chromium atoms [20] will allow experiments where the interaction varies from only contact to purely dipolar. In this way, many interesting predicted dipolar phenomena should be observed. Among them are, for instance, the stability of the ground state of a dipolar BEC [23-25] or its excitation spectrum [26]. Note that it has been recently suggested in Refs. $[27,28]$ that the $s$-wave scattering length $a$ could strongly depend on the dipole moment. However, there it has also been shown that for dipolar interaction strengths, which are not larger than the s-wave scattering strength, the latter is only rescaled by a moderate factor and remains positive. For the calculations in the present work we assume that this condition is fulfilled.

In this letter, we investigate how the critical temperature of a dipolar BEC depends on the dipole-dipole interaction. Consider an atomic gas trapped in a cylindersymmetric harmonic potential

$$
V(\mathbf{x})=\frac{M}{2}\left[\omega_{\perp}^{2}\left(x^{2}+y^{2}\right)+\omega_{\|}^{2} z^{2}\right]
$$

whose dipole moments $\mathbf{m}$ have the angle $\alpha$ with the $z$ axis, i.e. $\mathbf{m}=m(\sin \alpha, 0, \cos \alpha)$. In particular, we are 

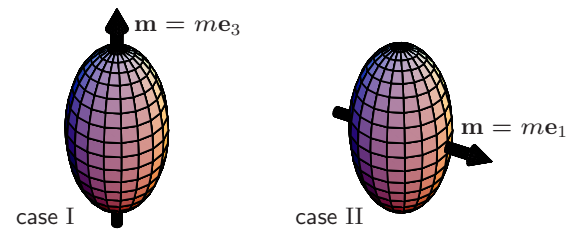

FIG. 1: Symmetry axes of harmonic trap (2) and two-particle interaction (1) are parallel and perpendicular in configuration I and II.

interested in the two extreme configurations I and II where the symmetry axis of the dipole moments is parallel $(\alpha=0)$ and perpendicular $(\alpha=\pi / 2)$ to the symmetry axis of the harmonic trap (2), respectively. Figure 1 illustrates the two cases for $\omega_{\|}<\omega_{\perp}$, where the atomic dipole moments lead to a residual attractive and repulsive interaction, respectively. At first we show that the corresponding critical temperature in configuration I and II is shifted above and below the value of a pure contact interaction. Subsequently we suggest to determine the difference of the critical temperatures in both configurations I and II to cancel out the influence of the isotropic contact interaction. We shall estimate which experimental parameters allow us to enhance this signal. For a pure contact interaction, the harmonic trap suppresses longwavelength fluctuations, so the leading shift of the critical temperature can be calculated perturbatively (see, for instance, the recent work [29] and the references cited therein). We expect that this reasoning also holds for our model interaction (1). Although the dipolar interaction is nonlocal, its scaling properties are the same as for a contact interaction.

In this letter we apply Feynman's diagrammatic technique of many-body theory $[30,31]$ and expand the grand-canonical free energy as

$$
\mathcal{F}=\mathcal{F}^{(0)}-\frac{1}{\beta}\left\{\frac{1}{2} \bigcirc \bigcirc+\frac{1}{2} \circlearrowleft+\ldots\right\} .
$$

The first term denotes the contribution of an ideal Bose gas at temperature $T=1 / k_{B} \beta$ where the harmonic trap potential (2) is treated semiclassically [32]

$$
\mathcal{F}^{(0)}=-\frac{1}{\beta(\hbar \beta \tilde{\omega})^{3}} \zeta_{4}\left(e^{\beta \mu}\right) .
$$

It contains the chemical potential $\mu$, the geometric mean of the trap frequencies $\tilde{\omega}=\left(\omega_{\|} \omega_{\perp}^{2}\right)^{1 / 3}$, and the polylogarithmic function $\zeta_{a}(z)=\sum_{n=1}^{\infty} z^{n} / n^{a}$. The two diagrams in Eq. (3) represent the direct and the exchange vacuum contribution, respectively, and have to be evaluated according to the Feynman rules: a straight line with an arrow represents the semiclassical interaction-free correlation function:

$$
\begin{aligned}
\mathbf{x}, \tau & -\mathbf{x}^{\prime}, \tau^{\prime} \equiv \int \frac{d^{3} p}{(2 \pi \hbar)^{3}} e^{i \mathbf{p}\left(\mathbf{x}-\mathbf{x}^{\prime}\right) / \hbar} \\
& \times \sum_{m=-\infty}^{\infty} \frac{e^{-i \omega_{m}\left(\tau-\tau^{\prime}\right)}}{\beta\left[-i \hbar \omega_{m}+\frac{\mathbf{p}^{2}}{2 M}+V\left(\frac{\mathbf{x}+\mathbf{x}^{\prime}}{2}\right)-\mu\right]}
\end{aligned}
$$

where $\omega_{m}=2 \pi m / \hbar \beta$ denotes the Matsubara frequency. The two-particle interaction potential enters via the diagram

$$
\text { x. }{ }^{\tau} \mathbf{x}^{\prime} \equiv \frac{-1}{\hbar} \int_{0}^{\hbar \beta} d \tau \int d^{3} x \int d^{3} x^{\prime} V^{(\mathrm{int})}\left(\mathbf{x}-\mathbf{x}^{\prime}\right)
$$

The grand-canonical free energy (3) is studied as a function of temperature $T$ for fixed particle number $N=$ $-\partial \mathcal{F} / \partial \mu$.

The phase transition, where a macroscopic occupation of the ground state sets in, occurs when the correlation function of the system diverges. From Eq. (5) we can see that this happens in the interaction-free case at $\mu_{c}^{(0)}=0$, as the divergence appears at the minimum of the trap potential $V(\mathbf{x})$ for vanishing Matsubara frequency $\omega_{m}$ and momentum p. In the presence of a 2-particle interaction, the full correlation function follows from a formula similar to (5) where the chemical potential $\mu$ is shifted by the self-energy: $\mu \rightarrow \mu+\hbar \Sigma\left(\mathbf{p}, \omega_{m} ; \mathbf{x}\right)$. It is defined by the Fourier-Matsubara transformed

$$
\int_{0}^{\hbar \beta} d \tau \int d^{3} x^{\prime} e^{i \omega_{m} \tau-i \mathbf{p} \mathbf{x}^{\prime} / \hbar} \Sigma\left(\mathbf{x}+\frac{\mathbf{x}^{\prime}}{2}, \tau ; \mathbf{x}-\frac{\mathbf{x}^{\prime}}{2}, 0\right)
$$

of the Feynman diagrams

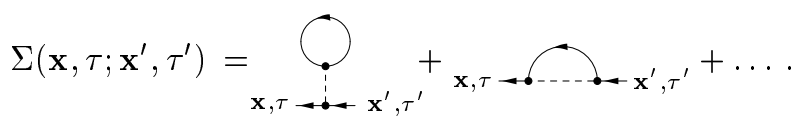

The critical chemical potential reads now [33]

$$
\mu_{c}=\min _{\mathbf{x}, \mathbf{p}}\left[\frac{\mathbf{p}^{2}}{2 M}+V(\mathbf{x})-\hbar \Sigma(\mathbf{p}, 0 ; \mathbf{x})\right],
$$

which leads to $\mu_{c}=-\hbar \Sigma(\mathbf{0}, 0 ; \mathbf{0})$ up to first order. Thus, evaluating the particle number $N=N(\mu)$ at the critical chemical potential $\mu=\mu_{c}$ yields the following first-order shift of the critical temperature with respect to the interaction-free critical temperature $T_{c}^{(0)}=$ $\hbar \tilde{\omega}[N / \zeta(3)]^{1 / 3} / k_{B}$ :

$$
\frac{\Delta T_{c}}{T_{c}^{(0)}}=-\frac{c_{\delta} a}{\lambda_{c}^{(0)}}+\left[3 \cos ^{2} \alpha-1\right] f\left(\frac{\omega_{\|}}{\omega_{\perp}}\right) \frac{\mu_{0} m^{2} M c_{\delta}}{48 \pi \hbar^{2} \lambda_{c}^{(0)}} .
$$

Here $\zeta(a)=\sum_{n=1}^{\infty} 1 / n^{a}$ is the Riemann zeta function and $\lambda=\left(2 \pi \hbar^{2} \beta / M\right)^{1 / 2}$ the thermodynamic de Broglie wave length. The dimensionless prefactor $c_{\delta}$ for the $\delta$ interaction has the value

$$
c_{\delta}=\frac{4}{3 \zeta(3)}\left[\zeta\left(\frac{3}{2}\right) \zeta(2)-\zeta\left(\frac{1}{2}, \frac{3}{2}, \frac{3}{2}\right)\right] \approx 3.426
$$

with the generalized Riemann zeta function $\zeta(a, b, c)=$ $\sum_{n=1}^{\infty} \sum_{n^{\prime}=1}^{\infty} 1 / n^{a} n^{\prime b}\left(n+n^{\prime}\right)^{c}$, whereas the dimensionless prefactor $f(\kappa)$ for the dipole-dipole interaction is a function of the ratio of the trap frequencies $\kappa=\omega_{\|} / \omega_{\perp}$ :

$$
f(\kappa)=\left\{\begin{array}{cc}
\frac{2 \kappa^{2}+1}{1-\kappa^{2}}-\frac{3 \kappa^{2} \operatorname{artanh} \sqrt{1-\kappa^{2}}}{\left(1-\kappa^{2}\right)^{3 / 2}} & , \kappa \neq 1 \\
0 & , \kappa=1 .
\end{array}\right.
$$



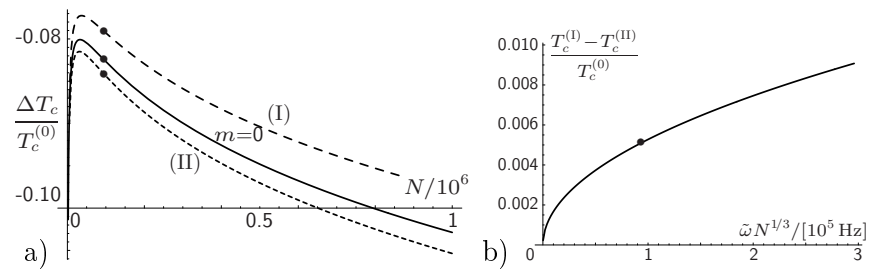

FIG. 2: a) Shift of the critical temperature $\Delta T_{c}$ with respect to the interaction-free critical temperature $T_{c}^{(0)}$ for a ${ }^{52} \mathrm{Cr}$ gas in a harmonic trap with frequencies $\omega_{\|}=2 \pi \cdot 138 \mathrm{~Hz}$ and $\omega_{\perp}=$ $2 \pi \cdot 486 \mathrm{~Hz}$ : without (straight line) and with (dashed lines) magnetic dipole-dipole interaction for the configurations I and II of Figure 1. b) Difference of the temperature shifts increases with particle number $N$ and geometric mean frequency $\tilde{\omega}$. The respective dots indicate the parameters of the Stuttgart ${ }^{52} \mathrm{Cr}$ experiment.

This function was already used in Ref. [19] to describe the mean-field magnetic dipole-dipole energy for a cylindrically symmetric BEC. Note that $f(\kappa)$ tends asymptotically to -2 for $\kappa \rightarrow \infty$ and 1 for $\kappa \rightarrow 0$, respectively. The general physical implications of our first-order perturbative result (10) are as follows. Without the dipoledipole interaction, i.e. at $m=0$, the critical temperature is shifted downwards with the dimensionless prefactor (11). This result for the isotropic contact interaction was originally derived in Ref. [34] within a mean-field approach and confirmed experimentally by investigating the onset of Bose-Einstein condensation in the hyperfine ground state of ${ }^{87} \mathrm{Rb}[35]$. Our result (10) for $m \neq 0$ shows that the critical temperature is increased in configuration I $(\alpha=0)$ twice as much as decreased in configuration II $(\alpha=\pi / 2)$ of Figure 1 for the trap anisotropy $\omega_{\|}<\omega_{\perp}$, due to the dipole-dipole interaction. Of course, changing the angle $\alpha$ allows us to tune the dipolar effect between these maximal and minimal values. In particular, we read off from Eq. (10) that the dipolar shift of the critical temperature vanishes for the magic angle $\alpha_{0}=\arccos (1 / \sqrt{3})=54.7^{\mathrm{O}}[22]$.

Now we discuss the consequences of our results for the ongoing experiments on the Bose-Einstein condensation of ${ }^{52} \mathrm{Cr}$-atoms at the University of Stuttgart, where the trap frequencies are $\omega_{1}=2 \pi \cdot 581 \mathrm{~Hz}, \omega_{2}=2 \pi \cdot 406 \mathrm{~Hz}$, $\omega_{3}=2 \pi \cdot 138 \mathrm{~Hz}$, so that the geometric mean frequency is $\tilde{\omega}=2 \pi \cdot 319 \mathrm{~Hz}$. The total number of atoms is $N=100000$, yielding an interaction-free critical temperature of about $T_{c}^{(0)}=670 \mathrm{nK}$. The corresponding finite-size correction was calculated in Refs. [36, 37]

$$
\left(\frac{\Delta T_{c}}{T_{c}^{(0)}}\right)_{F S}=-\frac{\zeta(2) \bar{\omega}}{2 \zeta^{2 / 3}(3) \tilde{\omega} N^{1 / 3}}
$$

where $\bar{\omega}$ is the arithmetic mean of the trap frequencies $\bar{\omega} \approx\left(\omega_{1}+\omega_{2}+\omega_{3}\right) / 3=2 \pi \cdot 375 \mathrm{~Hz}$. Thus, the finite-size correction of $T_{c}^{(0)}$ in the Stuttgart experiment amounts to $-1.8 \%$. This is to be compared with a shift of the critical temperature due to the contact and the magnetic dipole-dipole interaction following from formula (10).

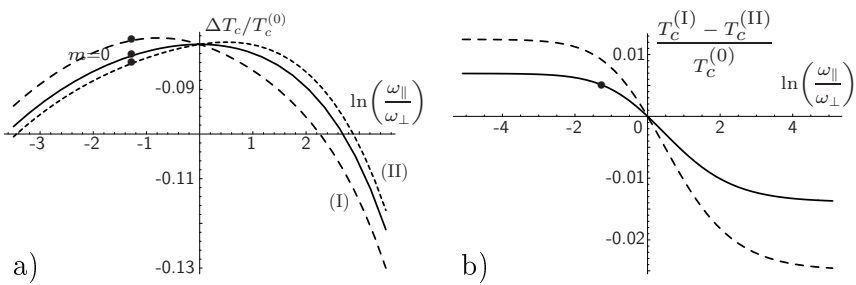

FIG. 3: a) Shift of the critical temperature $\Delta T_{c}$ with respect to the interaction-free critical temperature $T_{c}^{(0)}$ for $N=10^{5}$ ${ }^{52} \mathrm{Cr}$-atoms gas versus anisotropy parameter $\omega_{\|} / \omega_{\perp}$ of the harmonic trap without (straight line) and with (dashed lines) magnetic dipole-dipole interaction for the configurations I and II of Figure 1. b) Difference of the temperature shifts versus anisotropy parameter $\omega_{\|} / \omega_{\perp}$ for $\tilde{\omega} N^{1 / 3}=0.93 \cdot 10^{5} \mathrm{~Hz}$ (solid line) and $\tilde{\omega} N^{1 / 3}=3 \cdot 10^{5} \mathrm{~Hz}$ (dashed line). The respective dots indicate the parameters of the Stuttgart ${ }^{52} \mathrm{Cr}$ experiment.

The s-wave scattering length of the ${ }^{52} \mathrm{Cr}$-atoms is $a=105 a_{B}[20]$, i.e. roughly $2 \%$ of the thermodynamic de Broglie wave length $\lambda_{c}^{(0)}=5598 a_{B}$. The corresponding downwards shift of the critical temperature amounts then to $6.4 \%$. This is modified by the dipole-dipole interaction depending on the experimental set-up. In the Stuttgart experiment, the trap potential has actually three different frequencies but, since $\omega_{1}$ and $\omega_{2}$ are not far apart, we may identify $\omega_{\perp}=\sqrt{\omega_{1} \omega_{2}}=2 \pi \cdot 486 \mathrm{~Hz}$ and $\omega_{\|}=\omega_{3}$, yielding $\omega_{\|} / \omega_{\perp}=0.284$. In configuration I we obtain from (12) the result $f\left(\omega_{\|} / \omega_{\perp}\right)=0.733$, which leads via Eq. (10) to an extra upward shift of the critical temperature by $0.34 \%$ due to the magnetic dipole-dipole interaction. The downward shift in configuration II is half as big. Figure 2a plots the resulting total shift of the critical temperature $\Delta T_{c}$ for the ${ }^{52} \mathrm{Cr}$ gas with respect to the interaction-free critical temperature $T_{c}^{(0)}$ versus the particle number $N$. Both the finite-size corrections and the contact interaction lead to the main shift, on top of which the small dipolar effect is seen. Figure 3a shows how the same shifts depend for $N=10^{5}$ chromium atoms on the anisotropy parameter $\omega_{\|} / \omega_{\perp}$. The directions of the shifts change sign at the isotropy point $\omega_{\|}=\omega_{\perp}$.

The above results suggest to plot the difference between the critical temperatures of the two configurations I and II. This eliminates the isotropic effects of both the contact interaction and the finite-size correction, thus it exhibits most clearly the magnetic dipole-dipole interaction. For $N=10^{5}$ and $\tilde{\omega}=2 \pi \cdot 319 \mathrm{~Hz}$, the difference amounts to a net effect of $0.51 \%$ of the interaction-free critical temperature $T_{c}^{(0)}$. One possibility to enhance the difference of the critical temperatures is to choose a convenient anisotropy strength $\omega_{\|} / \omega_{\perp}$ of the harmonic trap potential as seen in Figure 3b. Furthermore, this dipolar effect increases with the total atom number $N$ and the geometric mean frequency $\tilde{\omega}$ as shown in Figure $2 \mathrm{~b}$, which therefore needs to be as large as possible. An experimentally feasible increase of the particle density in the trap could lead to a dipolar effect of more than $2 \%$, 
see dashed curve of Figure 3b.

At present, the best experiments which measure the critical temperature of a Bose-Einstein condensate involve error bars of $5 \%$ which represent the total systematic and statistical errors $[4,35]$. The systematic errors, however, can be eliminated by our suggestion to measure the difference of two critical temperatures. We expect that the remaining statistical errors can be reduced to $1 \%$ by averaging both atom number and critical temperature over many measurements.

So far, the dipolar nature of the chromium BEC has only been resolved in expansion experiments [21]. The analysis of this letter shows that it could be possible to detect a signal of the underlying magnetic dipole-dipole interaction also by measuring the critical temperature. Furthermore, our results will be useful for other dipolar systems with a tunable dipole moment, like heteronuclear molecules in low vibrational states [12-16], where the dipolar effect will be larger.

We thank A. Berra and S. Kling for discussions and DFG Priority Program SPP 1116 as well as SFB/TR 21.
[1] M.H. Anderson, J.R. Ensher, M.R. Matthews, C.E. Wieman, and E.A. Cornell, Science 269, 198 (1995)

[2] K.B. Davis, M.-O. Mewes, M.R. Andrews, N.J. van Druten, D.S. Durfee, D.M. Kurn, and W. Ketterle, Phys. Rev. Lett. 75, 3969 (1995)

[3] M. Greiner, O. Mandel, T. Esslinger, T.W. Hänsch, and I. Bloch, Nature 415, 39 (2002)

[4] A. Griesmaier, J. Werner, S. Hensler, J. Stuhler and T. Pfau, Phys. Rev. Lett. 94, 160401 (2005)

[5] R. deCarvalho, J.M. Doyle, B. Friedrich, T. Guillet, J. Kim, D. Patterson, and J.D. Weinstein, Eur. Phys. J. D 7, 289 (1999)

[6] J.J. McClelland and J.L. Hanssen, Phys. Rev. Lett. 96, 143005 (2006)

[7] L. Santos, G.V. Shlyapnikov, P. Zoller, and M. Lewenstein, Phys. Rev. Lett. 85, 1791 (2000)

[8] K. Afrousheh, P. Bohlouli-Zanjani, J.D. Carter, A. Mugford, and J.D.D. Martin, Phys. Rev. A 73, 063403 (2006)

[9] S. Yi and L. You, Phys. Rev. A 63, 053607 (2001)

[10] K. Góral, K. Rzazewski, and T. Pfau, Phys. Rev. A 61, 051601(R) (2000)

[11] J.P. Martikainen, M. Mackie, and K.A. Suominen, Phys. Rev. A 64, 037601 (2001)

[12] J.D. Weinstein, R. deCarvalho, T. Guillet, B. Friedrich, and J.M. Doyle, Nature 395, 148 (1998)

[13] H.L. Bethlem, G. Berden, and G. Meijer, Phys. Rev. Lett. 83, 1558 (1999)

[14] J.M. Sage, S. Sainis, T. Bergeman, and D. DeMille, Phys. Rev. Lett. 94, 203001 (2005)

[15] M.W. Mancini, G.D. Telles, A.R.L. Caires, V.S. Bagnato, and L.G. Marcassa, Phys. Rev. Lett. 92, 133203 (2004)

[16] C. Ospelkaus, S. Ospelkaus, L. Humbert, P. Ernst, K. Sengstock, and K. Bongs, Phys. Rev. Lett. 97, 120402 (2006)

[17] A. Derevianko, Phys. Rev. A 67, 033607 (2003); Phys. Rev. A 72, 039901(E) (2005)

[18] S. Yi and L. You, Phys. Rev. Lett 92, 193201 (2004)

[19] S. Giovanazzi, A. Görlitz, and T. Pfau, J. Opt. B 5, S208 (2003)

[20] J. Werner, A. Griesmaier, S. Hensler, J. Stuhler, T. Pfau,
A. Simoni and E. Tiesinga, Phys. Rev. Lett. 94, 183201 (2005)

[21] J. Stuhler, A. Griesmaier, T. Koch, M. Fattori, T. Pfau, S. Giovanazzi, P. Pedri, and L. Santos, Phys. Rev. Lett. 95, 150406 (2005)

[22] S. Giovanazzi, A. Görlitz, and T. Pfau, Phys. Rev. Lett. 89, $130401(2002)$

[23] L. Santos, G.V. Shlyapnikov, P. Zoller, and M. Lewenstein, Phys. Rev. Lett 85, 1791 (2000)

[24] D.H.J. O'Dell, S. Giovanazzi, and C. Eberlein, Phys. Rev. Lett. 92, 250401 (2004)

[25] C. Eberlein, S. Giovanazzi, and D.H.J. O'Dell, Phys. Rev. A 71, 033618 (2005)

[26] L. Santos, G.V. Shlyapnikov, and M. Lewenstein, Phys. Rev. Lett. 90, 250403 (2002)

[27] S. Ronen, D.C.E. Bortolotti, D. Blume, and J.L. Bohn, Phys. Rev. A 74, 033611 (2006)

[28] D.C.E. Bortolotti, S. Ronen, J.L. Bohn, and D. Blume, Phys. Rev. Lett. 97, 160402 (2006) and eprint: cond-mat/0604432

[29] O. Zobay, G. Metikas, and H. Kleinert, Phys. Rev. A 71, 043614 (2005)

[30] G.D. Mahan, Many-Particle Physics, Third Edition (Kluwer Academic/Plenum Publisher, New York, 2000)

[31] A. Pelster and K. Glaum, Phys. Stat. Sol. B 237, 72 (2003)

[32] H. Kleinert, Path Integrals in Quantum Mechanics, Statistics, Polymer Physics, and Financial Markets, Forth Edition (World Scientific, Singapore, 2006)

[33] K. Glaum and A. Pelster, eprint: cond-mat/0609374

[34] S. Giorgini, L.P. Pitaevskii, and S. Stringari, Phys. Rev. A 54, R4633 (1996)

[35] F. Gerbier, J.H. Thywissen, S. Richard, M. Hugbart, P. Bouyer, and A. Aspect, Phys. Rev. Lett. 92, 030405 (2004)

[36] S. Grossmann and M. Holthaus, Phys. Lett. A 208, 188 (1995)

[37] F. Dalfovo, S. Giorgini, L.P. Pitaevskii, and S. Stringari, Rev. Mod. Phys. 71, 463 (1999) 